\title{
Exact closed-form analytic wave functions in two dimensions: Contact-interacting fermionic spinful ultracold atoms in a rapidly rotating trap
}

\author{
Constantine Yannouleas $\mathbb{1}^{*}$ and Uzi Landman $\oplus^{\dagger}$ \\ School of Physics, Georgia Institute of Technology, Atlanta, Georgia 30332-0430, USA
}

(Received 12 January 2021; accepted 29 June 2021; published 30 July 2021)

\begin{abstract}
Exact two-dimensional analytic wave functions for an arbitrary number $N$ of contact-interacting lowestLandau-level (LLL) spinful fermions are derived with the use of combined numerical and symbolic computational approaches via analysis of exact Hamiltonian numerical diagonalization data. Closed-form analytic expressions are presented for two families of zero-interaction-energy states at given total angular momentum and total spin $0 \leqslant S \leqslant N / 2$ in the neighborhood of the $v=1$ filling, covering the range from the maximum density droplet to the first quasihole. Our theoretical predictions for higher-order spatial and momentum correlations reveal intrinsic polygonal, multi-ring crystalline-type structures, which can be tested with ultracold-atom experiments in rapidly rotating traps, simulating quantum Hall physics (including quantum LLL skyrmions).
\end{abstract}

DOI: 10.1103/PhysRevResearch.3.L032028

\section{INTRODUCTION}

Exact analytic solutions for the quantum many-body problem, whether in a closed-form algebraic expression or in the form of the Bethe ansatz, are highly coveted and soughtafter; however, they are available only for a few cases. Among this select group (for early pioneering studies see Refs. [1-7]), one-dimensional (1D) assemblies of strongly contact-interacting ultracold atoms have attracted much attention in the last few years [8-17], motivated by rapid experimental advances in the field of trapped ultracold atoms that allow direct verification of theoretical results. In this context, in-situ and time-of-flight single-atom measurements of real-space and momentum-space higher-order correlations, respectively, hold a great promise [18-31].

Here we derive closed-form exact analytic wave functions (EAWFs) for two-dimensional (2D) systems of spinful contact-interacting lowest-Landau-level (LLL) fermions that simulate fractional quantum Hall (FQH) physics [32-39] with trapped ultracold atoms. We first introduce an approach for the extraction of EAWFs from the digital information provided via numerical exact-diagonalization (i.e., the configuration interaction, CI [29,38-40]) of the many-body LLL Hamiltonian. Subsequently, we present illustrative examples, showing that such EAWFs exhibit intrinsic geometric structures [ultracold Wigner molecules (UCWMs)] in their higher-order correlations, in line with earlier findings using numerical CI solutions

\footnotetext{
*Constantine.Yannouleas@physics.gatech.edu

†Uzi.Landman@physics.gatech.edu
}

Published by the American Physical Society under the terms of the Creative Commons Attribution 4.0 International license. Further distribution of this work must maintain attribution to the author(s) and the published article's title, journal citation, and DOI. (see, e.g., Ref. [38]). The compact EAWFs enable consideration of larger assemblies compared to the CI-computed UCWMs [38].

Starting with the Laughlin trial wave function [41], compact algebraic forms have been extensively considered $[33,42-48]$ as approximations to the exact diagonalization solutions, both for electrons in semiconductors [42-46] and for ultracold bosons in rotating traps $[33,47,48]$. In several instances, like the Laughlin wave functions, it was shown that the variational trial functions $[33,44,46]$ may be exact solutions, with zero-interaction energy (OIE states), of specific short-range pseudopotential-type parent Hamiltonians $[33,44]$.

Because of the fermionic statistics, this paper relates to electronic 2D quantum LLL skyrmions [45,49-53], multicomponent quantum Hall systems [53], 2D anyons [37], and rotating electronic [54-57] and ultracold-atom [29,38,58] Wigner molecules. Experimental realization of such 2D systems (including bosonic analogs [34-37]) with a few ultracold fermionic atoms (e.g., $\left.{ }^{6} \mathrm{Li}\right)$ in rapidly rotating harmonic traps is currently pursued [39]. Importantly, unlike the skyrmion wave functions used in the literature [45,49-52], which are not eigenstates of the total spin (see particularly Ref. [51], the Appendix, and the Supplemental Material (SM) [59]), the EAWFs introduced here provide total-spin preserving symmetric polynomials for the quantum LLL skyrmions; for other spin-preserving polynomials (restricted to the spin-singlet state), see Ref. [60].

\section{METHODOLOGY}

Extensions of Girardeau's mapping between impenetrable bosons and noninteracting spinless fermions [1], and similar mappings $[8,10]$ applied to spinful and spin-parallel fermions, led to the formulation of a hard-core boundary condition for strongly repelling $1 \mathrm{D}$ fermions $[11,12]$. This entails vanishing 


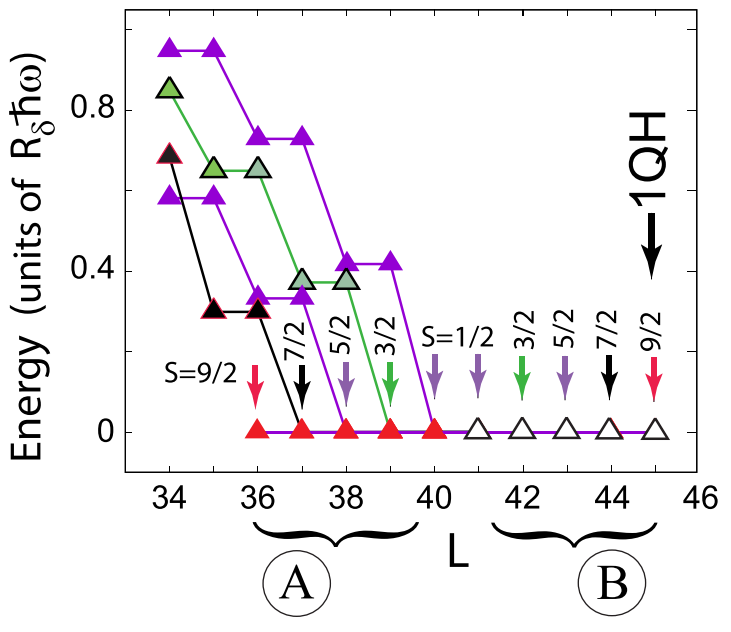

FIG. 1. CI-calculated, relative-ground-state LLL energy spectra for $N=9$ fermions associated with the contact-interaction term only; see third term of $H_{\mathrm{LLL}}$ in Eq. (1). Spectra in a given spin sector $S=$ $1 / 2,3 / 2,5 / 2,7 / 2$ and $S=9 / 2$ are explicitly denoted. The spectra were calculated for $S_{z}=1 / 2$; however, they are independent of the precise value of $S_{z}$. The 0IE states of family $A$ are colored in red; those of family $B$ are colored in white. The first quasihole state is also explicitly denoted. $L$ is the total angular momentum.

of the many-body wave functions when two fermions with antiparallel spins are at the same position (in addition to the vanishing for parallel spins due to the Pauli exclusion principle). Concomitant of this condition is the appearance of OIE states.

In $\mathrm{CI}$ calculations and for a given number $N$ of spinful LLL fermions, the 0IE states emerge in each spin sector $\left(S, S_{z}\right)$; see Fig. 1 for the case of $N=9$ LLL fermions interacting with a $\sum_{i<j} \delta\left(z_{i}-z_{j}\right)$ two-body potential, where $z_{i}=x_{i}+i y_{i}$ (with $i=1,2, \ldots, N$ ). Interest in such 0IE states arises from the following: (i) They can be prepared experimentally [39]. (For bosons, the experimental expectations for OIE states include fittingly the $v=1 / 2$ bosonic Laughlin state $[34,35,61]$.) (ii) They represent microscopic states that describe quantum LLL skyrmions $[45,50]$. (iii) The Laughlin states are 0IE eigenstates associated with short-range pseudopotential-type Hamiltonians $[44,62]$. (iv) For fully polarized fermions, OIE states have been associated with the gapless edge excitations of the Laughlin droplet [63] in extended semiconductor samples.

The many-body Hamiltonian describing ultracold neutral atoms in a rapidly rotating trap $[29,33-35,39,58]$ is given by

$$
\frac{H_{\mathrm{LLL}}}{\hbar \omega}=N+\left(1-\frac{\Omega}{\omega}\right) L+2 \pi R_{\delta} \sum_{i<j}^{N} \delta\left(z_{i}-z_{j}\right),
$$

where $\omega$ and $\Omega$ are, respectively, the parabolic trapping and rotational frequencies of the trap, and $L$ denotes the total angular momentum, $L=\sum_{i=1}^{N} l_{i}$, normal to the rotating-trap plane; the energies are in units of $\hbar \omega$ and the lengths in units of the oscillator length $\Lambda=\sqrt{\hbar /(M \omega)}$, with $M$ being the fermion mass. The first and second terms express the LLL kinetic energy, $H_{K}$, and the third term represents the contact interaction, $H_{\text {int }}$.
Our methodology integrating both numerical (e.g., FORTRAN) and symbolic (algebraic, e.g., MATHEMATICA [64]) languages consists of two steps: (1) numerical diagonalization of the Hamiltonian matrix problem employing the ARPACK solver $[65,66]$ of large-scale sparse eigenvalue problems, followed by step (2) where the numerically exact CI wave functions

$$
\Phi_{\mathrm{CI}}\left(z_{1} \sigma_{1}, \ldots, z_{N} \sigma_{N}\right)=\sum_{I} c_{\mathrm{CI}}(I) \Psi_{I}\left(z_{1} \sigma_{1}, \ldots, z_{N} \sigma_{N}\right)
$$

are analyzed and processed using symbolic scripts targeting extraction of the corresponding exact analytical wave functions.

The basis Slater determinants that span the Hilbert space are

$$
\Psi_{I}=\operatorname{Det}\left[\varphi_{j_{r}}\left(z_{s}\right) \sigma_{j_{r}}(s)\right] / \sqrt{N !},
$$

where $r, s=1, \ldots, N$, the LLL single-particle orbitals are

$$
\varphi_{j}(z)=z^{l_{j}} e^{-z z^{*} / 2} / \sqrt{\pi l_{j} !},
$$

and $\sigma$ signifies an up $(\alpha)$ or a down $(\beta)$ spin. The master index $I$ counts the number of ordered arrangements (lists) $\left\{j_{1}, j_{2}, \ldots, j_{N}\right\}$ under the restriction that $1 \leqslant j_{1}<$ $j_{2}<\cdots<j_{N} \leqslant K ; K \in \mathbb{N}$ is chosen large enough to provide numerical convergence. Below explicit mention of the Gaussian factor is omitted.

Step (2) starts with the rewriting of the CI wave function $\Phi_{\mathrm{CI}}$ in Eq. (2) as

$$
\Phi_{\mathrm{alg}}\left(z_{1} \sigma_{1}, \ldots, z_{N} \sigma_{N}\right)=\sum_{I} c_{\mathrm{alg}}(I) \Psi_{I}\left(z_{1} \sigma_{1}, \ldots, z_{N} \sigma_{N}\right),
$$

where the replacement of the subscript "CI" by "alg" corresponds to the fact that, using the symbolic language code, one obtains an equivalent multivariate homogeneous polynomial $\Phi_{\text {alg }}$ with algebraic coefficients $c_{\text {alg }}$; see the transcription of coefficients for $N=4$ and $N=9$ in Tables STI and STII in the SM [59].

Validation of our closed-form analytic wave functions (see below) is achieved via direct comparison of the numerical CI coefficients, $c_{\mathrm{CI}}$, with those in $\Phi_{\mathrm{alg}}$ [Eq. (5)], thus circumventing uncertainties, associated with the common use of wave function overlap $[39,41,43,44,46]$, due to the van Vleck-Anderson orthogonality catastrophe [67-73].

Invariably, the symbolic code is able to simplify the derived multivariate polynomial in Eq. (5) to the compact form of a product of a Vandermonde determinant (VDdet), $\prod_{i<j}^{N}\left(z_{i}-\right.$ $z_{j}$ ), involving the space coordinates only, with a symmetric polynomial (under two-particle exchange) with mixed space and spin coordinates [see Eq. (6) below]. The factoring out of the VDdet reflects the fact that $\Phi_{\text {alg }}$ represents a OIE LLL state.

Using symbolic scripts, we verify further that the fully algebraic $\Phi_{\text {alg }}$ [Eq. (6)] is indeed an eigenstate of the total spin, obeying the Fock condition [74]. The final closed form expressions [see Eq. (8) below] are derived for $N \leqslant 9$, but they are valid for any $N$, thus circumventing the CI numerical diagonalization of large matrices, which is not feasible for $N \geqslant \sim 10$.

For the CI diagonalization, a small perturbing term $V_{P}$ (e.g., a small trap deformation [38], or a small hard-wall boundary 
[75]) needs to be added to the LLL Hamiltonian in Eq. (1). This has a negligible influence on the numerical eigenvalues, but it is instrumental in lifting the degeneracies among the 0IE states and thus produces CI states whose total spin $S$ is a good quantum number.

\section{TARGETED TOTAL SPINS AND ANGULAR MOMENTA}

For each size $N$, we provide analytic expressions for the maximum-spin $\left(S=S_{z}\right)$ OIE ground states with angular momenta $L=L_{0}+\Delta L$ [with $L_{0}=N(N-1) / 2$ ] from $\Delta L=0$ (maximum density droplet) to $\Delta L=N$ (first quasihole, $1 \mathrm{QH}$ ); they form two families $A$ and $B$ (see Fig. 1 for an illustration).

Using $k$ to denote the number of spin-up fermions and $p$ that of spin-down fermions, and focusing on the case with $k \geqslant$ $p$ (or equivalently $p \leqslant N / 2$ ), the states in both families are associated with the same set of total spins specified as $S=$ $S_{z}=(k-p) / 2=N / 2-p$. Furthermore, given a pair $(k, p)$ :

(A) The states in family $A$ have $\Delta L=p$, with $\Delta L$ varying from 0 to $N / 2$ for even $N$, and from 0 to $(N-1) / 2$ for odd $N$.

(B) The states in family $B$ have $\Delta L=k$, with $\Delta L$ varying from $N / 2$ to $N$ for even $N$, and from $(N+1) / 2$ to $N$ for $\operatorname{odd} N$.

The states in family $A$ are unique ground states, whereas those in family $B$ are part of degenerate manifolds. (This degeneracy is lifted as described above.)

\section{THE EXACT OIE LLL WAVE FUNCTIONS}

\section{Mathematical preliminary}

The quantity $k$-subset $($ list $)$ is a subset containing exactly $k$ elements out of the set of $n$ elements (named list). The number of $k$-subsets on $n$ elements is given by $\left(\frac{n}{k}\right)=\frac{n !}{k !(n-k) !}$. The set represented by list is taken to be a list of cardinally ordered positive integers. For example, there are 62 -subsets when list $=\{1,2,3,4\}$, namely, $\{1,2\},\{1,3\},\{1,4\},\{2,3\}$, $\{2,4\}$, and $\{3,4\}$.

\section{General form of the OIE LLL wave functions}

The compact algebraic expression has the general form

$$
\begin{aligned}
& \Phi_{\text {alg }}\left(z_{1} \chi(1), \ldots, z_{N} \chi(N)\right) \propto \Phi_{V}\left(l_{1}, \ldots, l_{N} ; z_{1}, \ldots, z_{N}\right) \\
& \quad \times \Phi_{\text {sym }}\left(z_{1} \chi(1), \ldots, z_{N} \chi(N)\right),
\end{aligned}
$$

where $\chi(i)$ denotes an up spin, $\alpha$, or down spin, $\beta$, and $i=$ $1, \ldots, N$.

$\Phi_{V}$ is a Vandermonde determinant,

$$
\Phi_{V}([l] ;[z])=\operatorname{Det}\left[z_{i}^{l_{j}}\right]=\prod_{i<j}^{N}\left(z_{i}-z_{j}\right),
$$

where $l_{j}=(j-1)$ and $i, j=1,2, \ldots, N$. The product of Jastrow factors above reflects the fact that the wave function in Eq. (6) is a OIE eigenstate of the contact-interaction term, $H_{\text {int }}$, in Eq. (1).

Due to the fermionic symmetry of the $\Phi_{\text {alg }}, \Phi_{\text {sym }}$ has to be symmetric under the exchange of any pair of indices $i$ and $j$.
Furthermore, $\Phi_{\text {sym }}$ can be written as

$$
\Phi_{\text {sym }}\left(z_{1} \chi(1), \ldots, z_{N} \chi(N)\right)=\sum_{m=1}^{K} \mathcal{P}_{m}^{o}[z] \mathcal{Z}_{m},
$$

where $\mathcal{P}_{m}^{o}$ (defined below) are homogeneous multivariate polynomials of order $o=p$ (family $A$ ) or $o=k$ (family $B$ ), and

$$
\mathcal{Z}_{m}=\alpha\left(i_{1}\right) \alpha\left(i_{2}\right) \cdots \alpha\left(i_{k}\right) \beta\left(j_{k+1}\right) \cdots \beta\left(j_{N}\right)
$$

is one of the $K=N ! /(k ! p !)$ distinct spin primitives having $k \leqslant N$ up and $p=N-k \leqslant k$ down spins. The set of indices $\left\{i_{1}, \ldots, i_{k}\right\}$ is the $m$ th element $(m=1,2, \ldots, K)$ of the $k$ subsets of the cardinal list (top-level list, see below) specified as list $=1,2, \ldots, N\}$. The set of indices $\left\{j_{k+1}, \ldots, j_{N}\right\}$ is complementary to the $\left\{i_{1}, \ldots, i_{k}\right\}$ set.

The $\Phi_{V}$ [Eq. (7)] corresponds to a filling factor $v=1$, whereas the filling fraction corresponding to Eq. (6) [with $\Phi_{\text {sym }}$ given in Eq. (8) through polynomials of order $o$ ] is near $v=1$. These fractions are indeed the ones most likely to be accessed first in upcoming experiments [39].

\section{Algebraic expressions for the polynomials $\mathcal{P}_{m}^{o}([z])$}

For each $S=S_{z}=(k-p) / 2$, except when $k=p$ which has a single state, there exists a pair of targeted LLL states, with one state of the pair belonging to family $A$ and the other to family $B$ (see Fig. 1 for an example).

Family A: First, the following square matrices of rank $p$ (the number of spin-down fermions) need to be considered:

$$
\mathbf{M}_{q, m}=\left[\begin{array}{ccc}
z_{i_{1}}-z_{j_{k+1}} & \ldots & z_{i_{1}}-z_{j_{N}} \\
\vdots & \ddots & \vdots \\
z_{i_{p}}-z_{j_{k+1}} & \ldots & z_{i_{p}}-z_{j_{N}}
\end{array}\right],
$$

where the dummy indices $i_{1}, \ldots, i_{p}$ here are associated with spin-up fermions, and the set $\left\{i_{1}, \ldots, i_{p}\right\}$ denotes the $q$ th subset among the $p$-subsets on a second-level list-2, with list -2 being the $m$ th element among the $k$-subsets on the $\{1,2, \ldots, N\}$ top-level list. The number of $p$-subsets of any second-level list-2 is $K_{2}=k ! /(p !(k-p) !)$, and thus the $q$ subscript runs from 1 to $K_{2}$. The set of indices $\left\{j_{k+1}, \ldots, j_{N}\right\}$ is complementary to the $\left\{i_{1}, \ldots, i_{k}\right\}$ set, and thus it remains constant for a given $m$ index in the matrices defined in Eq. (10). (Recall that $k$ is the total number of spin-up fermions, and that $\left\{i_{1}, \ldots, i_{k}\right\}$ is also referred to as a secondlevel list.)

The expression for the polynomial is given by

$$
\mathcal{P}_{m}^{p}([z])=\sum_{q=1}^{K_{2}} \operatorname{Perm}\left[\mathrm{M}_{q, m}\right],
$$

where the symbol "Perm" denotes a permanent.

The analytic expressions of the states with $S_{z}<S$, in a given spin multiplicity $2 S+1$, are obtained by repeated application of the spin lowering operator.

Example: We consider the state associated with $N=5$, $S=S_{z}=1 / 2$, and $L=12$. Note that $L_{0}=N(N-1) / 2=10$ in the corresponding fully polarized case. There are $K=$ $5 ! /(3 ! 2 !)=10$ spin primitives $\mathcal{Z}_{m}$, with $m=1,2, \ldots, 10$; they correspond to the ten 3 -subsets on the top-level 

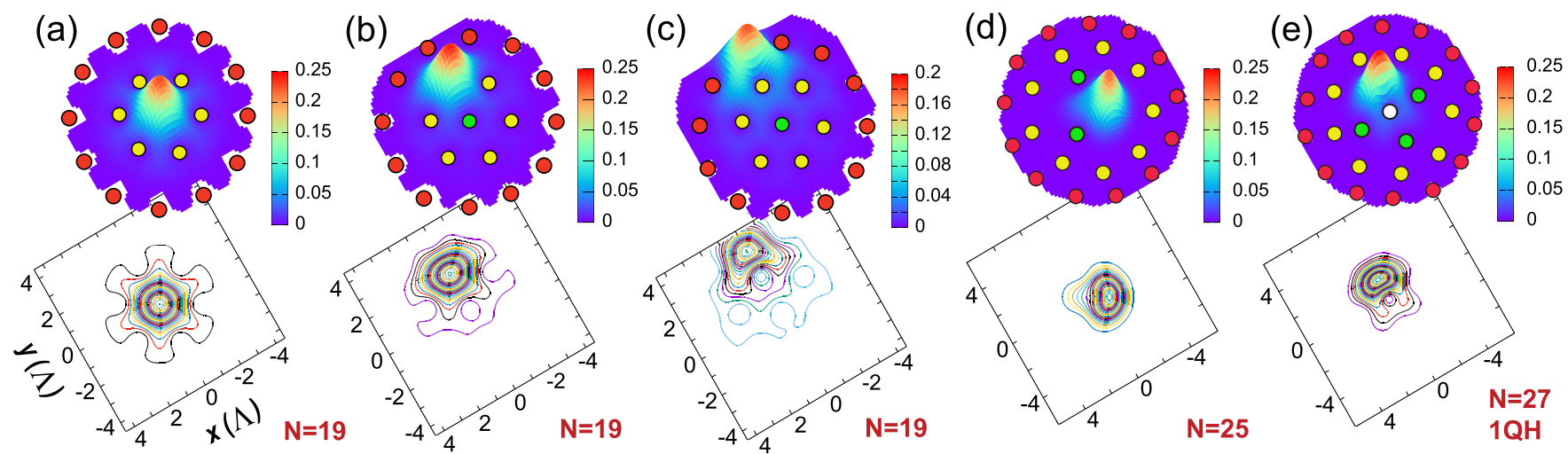

FIG. 2. $N$ th-order spin-unresolved correlations for $N$ LLL fermions. (a)-(c) $N=19$ with $L=180$, and $S=S_{z}=1 / 2$. (d) $N=25$ with $L=301$, and $S=S_{z}=23 / 2$. (e) $N=27$ with $L=378$, and $S=S_{z}=27 / 2$ (1QH state). Fixed fermions are marked by red dots for the outer ring, yellow dots for the middle ring, and green dots for the inner ring. The white dot for the $1 \mathrm{QH}$ state denotes the additional zero at the origin. Vertical axes: arbitrary units. See text for details. In the LLL, momentum correlations coincide with the spatial ones [38].

list $=\{1,2,3,4,5\}$, i.e., $\{1,2,3\}(m=1),\{1,2,4\}(m=2)$, $\{1,2,5\}(m=3),\{1,3,4\}(m=4),\{1,3,5\}(m=5),\{1,4,5\}$ $(m=6),\{2,3,4\}(m=7),\{2,3,5\}(m=8),\{2,4,5\}(m=$ $9),\{3,4,5\}(m=10)$.

Here $k=3, p=2$, and there are $K_{2}=3$ 2-subsets for each ( $m$ th) 3 -subset listed above. $K_{2}=3$ is also the number of permanents entering in expression (11), i.e., $q=1, \ldots, 3$. Choosing $m=10$ as an example, the three 2-subsets are $\{3,4\},\{3,5\}$, and $\{4,5\}$, and the three associated matrices $\mathbf{M}_{q, 10}$ are given by

$$
\mathbf{M}_{q, 10}=\left[\begin{array}{ll}
\eta(q, 1)-z_{1} & \eta(q, 1)-z_{2} \\
\eta(q, 2)-z_{1} & \eta(q, 2)-z_{2}
\end{array}\right]
$$

$$
\mathbf{M}_{m}^{B}=\left[\begin{array}{ccc}
z_{i_{1}}-z_{j_{k+1}} & \ldots & z_{i_{1}}-z_{j_{N}} \\
\vdots & \ddots & \vdots \\
z_{i_{k}}-z_{j_{k+1}} & \ldots & z_{i_{k}}-z_{j_{N}}
\end{array}\right.
$$

Above, the set of indices $\left\{i_{1}, \ldots, i_{k}\right\}$ is the $m$ th element of the $k$-subsets associated with the spin-up fermions [see Eq. (9)]. Because $k>p$, the complimentary set of the $p$ spin-down indices $\left\{j_{k+1}, \ldots, j_{N}\right\}$ has been expanded to contain exactly $k$ elements, through the introduction of virtual fermion coordinates such that $z_{j_{s}}=0$ for all $s>N$; see specific matrices $\mathbf{M}_{m}^{B}$, as well as a comparison with the wave functions in Ref. [45], in the Appendix and the SM [59].

Note that the first quasi-hole state $(1 \mathrm{QH})[41,75]$ coincides with the analytic expression associated with family $B$ above for $L=L_{0}+N$.

\section{HIGHER-ORDER CORRELATIONS}

We used the analytic wave functions above to calculate spin-unresolved higher-oder correlations for $N=19,25$, and 27 fermions; see Fig. 2 (for completeness, see Fig. SF1 for $N=9$ in the SM [59]). The $n$-body correlations for spinful fermions were defined in detail in Sec. II C of Ref. [38]. For the $N$-body $\Phi_{\text {alg }}$ [Eq. (6)], the spatial $n$-body correlation is with with $q=1,2,3 ; \eta(1,1)=z_{3}, \eta(1,2)=z_{4}, \eta(2,1)=$ $z_{3}, \eta(2,2)=z_{5}$, and $\eta(3,1)=z_{4}, \eta(3,2)=z_{5}$.

An additional example is presented in the SM [59].

Family B: Similarly, we found that the symmetric polynomials $\mathcal{P}_{m}^{k}([z])$ related to the ground states of family $B$ consist always [for any $m$ in the summation of Eq. (8)] of a single permanent associated with a matrix of rank $k$ (the number of spin-up fermions), amely,

$$
\mathcal{P}_{m}^{k}([z])=\operatorname{Perm}\left[\mathbf{M}_{m}^{B}\right]
$$

with

$$
\left.\begin{array}{ccc}
z_{i_{1}}-z_{j_{N+1}} & \ldots & z_{i_{1}}-z_{j_{2 k}} \\
\vdots & \ddots & \vdots \\
z_{i_{k}}-z_{j_{N+1}} & \cdots & z_{i_{k}}-z_{j_{2 k}}
\end{array}\right]
$$

given in a compact form by

$$
\begin{aligned}
\mathcal{G}^{n}(N)= & \left(1-\delta_{n, N}\right) \int\left|\Phi_{\mathrm{alg}}\left(z_{1} \chi(1), \ldots, z_{N} \chi(N)\right)\right|^{2} \\
& \times d z_{n+1} d \chi(n+1) \cdots d z_{N} d \chi(N)+\delta_{n, N}\left|\Phi_{\mathrm{alg}}\right|^{2},
\end{aligned}
$$

with $n=2, \ldots, N . \mathcal{G}^{n}(N)$ gives the conditional probability to find particles $n, \ldots, N$ anywhere, for prespecified (fixed) locations of particles $1, \ldots, n-1$ with predetermined (resolved) or unspecified (unresolved) spins.

For $N=19$, Figs. 2(a), 2(b), and 2(c) display structured $N$ th-order correlations for the spin state with $S=$ $S_{z}=1 / 2$ and total angular momentum $L=180$. Extending Ref. [38], we found similar crystalline structures also in the $N$ th-order correlations of the associated fully polarized, single VDdet state with $S=19 / 2, S_{z}=19 / 2$, and $L_{0}=171$ (Pauli-exclusion-only case, experimentally investigated [28]). Figure 2(d) displays the structured $N$ th-order correlation for $N=25$ with $L=301$ and $S=S_{z}=23 / 2$, whereas Fig. 2(e) 
presents the structured $N$ th-order correlations for the $1 \mathrm{QH}$ for $N=27$ (with $L=378$ and $S=S_{z}=27 / 2$ ). The implied intrinsic geometric structure (UCWM) in Fig. 2 is a polygonal triple ring $\left(n_{1}, n_{2}, n_{3}\right)$ of localized fermions (with $n_{1}+$ $\left.n_{2}+n_{3}=N\right)$; specifically $(1,6,12)$ for Figs. 2(a), 2(b), 2(c), $(3,9,13)$ for Fig. $2(d)$, and $(4,9,14)$ for Figs. 2(e). We note that in the LLL neighborhood of $v=1$ (expected in experiments with trapped ultracold fermions [39]), the intrinsic ring geometry can be probed only with higher-order correlations. Indeed in this case, the second-order correlations are structureless; see the findings for $N=4[(0,4)$ single ring $]$ and $N=6[(1,5)$ double ring] in Ref. [38].

\section{CONCLUSION}

An approach for deriving exact closed-form analytic expressions for the wave functions (beyond the Jastrow-factors paradigm) of an assembly of 2D contact-interacting spinful LLL fermions (for any $N$ ) was introduced and validated. Such expressions require as input only the three parameters $N$ (number of particles), $L$ (total angular momentum), and $S$ (total spin). Examples were presented for two families of zero-interaction-energy states, from the maximum density droplet to the first quasihole in the neighborhood of $v=1$. Ensuing theoretical predictions for higher-order momentum correlations for $N=19,25$, and 27, revealing intrinsic polygonal, multiring crystalline configurations, could be tested with ultracold-atom experiments in rotating traps simulating spinful quantum Hall physics, including LLL skyrmions. The present approach can be extended to the neighborhood of any $v=1 / m$ that starts with a Laughlin wave function.

\section{ACKNOWLEDGMENTS}

This work has been supported by a grant from the Air Force Office of Scientific Research (AFSOR) under Award No. FA9550-21-1-0198. Calculations were carried out at the GATECH Center for Computational Materials Science.

\section{APPENDIX: COMPARISON WITH THE SYMMETRIC POLYNOMIALS FOR QUANTUM SKYRMIONS IN REF. [45]}

We compare here with the symmetric polynomials for the seed skyrmions specified in Eq. (6) of Ref. [45] or Eq. (2) in Ref. [49].
Omitting the trivial Gaussian functions, these polynomials are given by the single formula

$$
\Phi_{p}^{\mathrm{sk}, \mathrm{MFB}}=\sum_{m}^{K} z_{i_{1}} z_{i_{2}} \cdots z_{i_{k}} \mathcal{Z}_{m},
$$

where $\mathcal{Z}_{m}$ are the spin primitives defined in Eq. (9), and the superscript MFB stands for MacDonald-Fertig-Brey. The subscript $m$ runs over the $k$-subsets $\left\{i_{1}, \ldots, i_{k}\right\}$ of the list $=\{1,2,3, \ldots, N\}, k=N \uparrow$ being the number of spin-up fermions, with $p=N-k=N \downarrow$ being that of the spin-down fermions. The number of $k$-subsets is $K=N ! /(k ! p !)$. As is the case in Ref. [45], one can take the index $m$ as running over the $p$-subsets associated with the spin-down fermions, because there is a one-to-one correspondence to the $k$-subsets of the spin-up fermions. Note that Ref. [45] (Ref. [49]) uses the capital letter $K(R)$ in place of our $p$.

We consider the case $N=5, k=4, p=1, S=S_{z}=3 / 2$, and $\Delta L=4$, belonging to family $B$ in our exposition.

According to Eq. (A1), the corresponding MFB symmetric polynomial is

$$
\begin{aligned}
\Phi_{p=1}^{\mathrm{sk}, \mathrm{MFB}}= & z_{1} z_{2} z_{3} z_{4} \mathcal{Z}_{1}+z_{1} z_{2} z_{3} z_{5} \mathcal{Z}_{2}+z_{1} z_{2} z_{4} z_{5} \mathcal{Z}_{3} \\
& +z_{1} z_{3} z_{4} z_{5} \mathcal{Z}_{4}+z_{2} z_{3} z_{4} z_{5} \mathcal{Z}_{5} .
\end{aligned}
$$

The corresponding exact symmetric polynomial derived in this paper is given by Eqs. (8) and (13), namely,

$$
\Phi_{\text {sym }}^{\text {exact }}(N=5, N \uparrow=4, \Delta L=4)=\sum_{m=1}^{5} \mathcal{P}_{m}^{4}[z] \mathcal{Z}_{m},
$$

Expanding the permanents, one obtains for the space-only polynomials $\mathcal{P}_{m}^{4}[z]$ above (with $m=1, \ldots, 5$, in front of the $\mathcal{Z}_{m}$ spin primitives):

$$
\begin{aligned}
\mathcal{P}_{m}^{4}[z]= & c_{1} z_{1} z_{2} z_{3} z_{4}+c_{2} z_{1} z_{2} z_{3} z_{5}+c_{3} z_{1} z_{2} z_{4} z_{5} \\
& +c_{4} z_{1} z_{3} z_{4} z_{5}+c_{5} z_{2} z_{3} z_{4} z_{5},
\end{aligned}
$$

with $c_{i}=4$ when $i=m$ and $c_{i}=-1$ otherwise.

The polynomial in Eq. (A3) is clearly different from the MFB one [Eq. (A2)]. We verified that the wave functions derived here are eigenfunctions of the square, $\hat{S}^{2}$, of the totalspin operator (with eigenvalue $15 / 4$ and $S=3 / 2$ for the case in this Appendix), whereas the MFB ones are not (see also Ref. [51]); for details see Ref. [59].
[1] M. Girardeau, Relationship between systems of impenetrable bosons and fermions in one dimension, J. Math. Phys. 1, 516 (1960).

[2] E. H. Lieb and W. Liniger, Exact analysis of an interacting Bose gas. I. The general solution and the ground state, Phys. Rev. 130, 1605 (1963); M. Gaudin, Un système à une dimension de fermions en interaction, Phys. Lett. A 24, 55 (1967); C. N. Yang, Some Exact Results for the Many-Body Problem in One Dimension with Repulsive Delta-Function Interaction, Phys. Rev. Lett. 19, 1312 (1967).

[3] J. B. McGuire, Study of exactly soluble one-dimensional $\mathrm{N}$ body problems, J. Math. Phys. 5, 622 (1964).
[4] F. Calogero, Solution of the one-dimensional $N$-body problem with quadratic and/or inversely quadratic pair potentials, J. Math. Phys. 12, 419 (1971); Erratum: Solution of the onedimensional $N$-body problems with quadratic and/or inversely quadratic pair potentials [J. Math. Phys. 12, 419-436 (1971)] 37, 3646(E) (1996).

[5] B. Sutherland, Exact results for a quantum many-body problem in one dimension, Phys. Rev. A 4, 2019 (1971).

[6] D. C. Mattis, The Many-Body Problem: An Encyclopedia of Exactly Solved Models in One Dimension (World Scientific, Singapore, 1993). 
[7] B. Sutherland, Beautiful Models: 70 Years of Exactly Solved Quantum Many-Body Problems (World Scientific, Singapore, 2004).

[8] M. D. Girardeau, E. M. Wright, and J. M. Triscari, Ground-state properties of a one-dimensional system of hard-core bosons in a harmonic trap, Phys. Rev. A 63, 033601 (2001).

[9] M. D. Girardeau and A. Minguzzi, Soluble Models of Strongly Interacting Ultracold Gas Mixtures in Tight Waveguides, Phys. Rev. Lett. 99, 230402 (2007).

[10] M. D. Girardeau, Two super-Tonks-Girardeau states of a trapped one-dimensional spinor Fermi gas, Phys. Rev. A 82, 011607(R) (2010)

[11] L. Guan, S. Chen, Y. Wang, and Z.-Q. Ma, Exact Solution for Infinitely Strongly Interacting Fermi Gases in Tight Waveguides, Phys. Rev. Lett. 102, 160402 (2009).

[12] X. Cui and T-L Ho, Ground-state ferromagnetic transition in strongly repulsive one-dimensional Fermi gases, Phys. Rev. A 89, 023611 (2014).

[13] F. Deuretzbacher, D. Becker, J. Bjerlin, S. M. Reimann, and L. Santos, Quantum magnetism without lattices in strongly interacting one-dimensional spinor gases, Phys. Rev. A 90, 013611 (2014).

[14] A. G. Volosniev, D. V. Fedorov, A. S. Jensen, M. Valiente, and N. T. Zinner, Strongly interacting confined quantum systems in one dimension, Nat. Commun. 5, 5300 (2014).

[15] J. Levinsen, P. Massignan, G. M. Bruun, and M. M. Parish, Strong-coupling ansatz for the one-dimensional Fermi gas in a harmonic potential, Sci. Adv. 1, e1500197 (2015).

[16] L. Yang and X. Cui, Effective spin-chain model for strongly interacting one-dimensional atomic gases with an arbitrary spin, Phys. Rev. A 93, 013617 (2016).

[17] M. Beau, S. M. Pittman, G. E. Astrakharchik, and A. del Campo, Exactly Solvable System of One-Dimensional Trapped Bosons with Short- and Long-Range Interactions, Phys. Rev. Lett. 125, 220602 (2020).

[18] M. Greiner, O. Mandel, T. Esslinger, T. W. Hänsch, and I. Bloch, Quantum phase transition from a superfluid to a Mott insulator in a gas of ultracold atoms, Nature (London) 415, 39 (2002).

[19] F. Gerbier, A. Widera, S. Fölling, O. Mandel, T. Gericke, and I. Bloch, Phase Coherence of an Atomic Mott Insulator, Phys. Rev. Lett. 95, 050404 (2005).

[20] F. Gerbier, A. Widera, S. Fölling, O. Mandel, T. Gericke, and I. Bloch, Interference pattern and visibility of a Mott insulator, Phys. Rev. A 72, 053606 (2005).

[21] A. Omran, M. Boll, T. A. Hilker, K. Kleinlein, G. Salomon, I. Bloch, and Ch. Gross, Microscopic Observation of Pauli Blocking in Degenerate Fermionic Lattice Gases, Phys. Rev. Lett. 115, 263001 (2015).

[22] H. Ott, Single atom detection in ultracold quantum gases: A review of current progress, Rep. Prog. Phys. 79, 054401 (2016).

[23] S. S. Hodgman, R. I. Khakimov, R. J. Lewis-Swan, A. G. Truscott, and K. V. Kheruntsyan, Solving the Quantum ManyBody Problem Via Correlations Measured with a Momentum Microscope, Phys. Rev. Lett. 118, 240402 (2017).

[24] H. Cayla, C. Carcy, Q. Bouton, R. Chang, G. Carleo, M. Mancini, D. Clément, Single-atom-resolved probing of lattice gases in momentum space, Phys. Rev. A 97, 061609(R) (2018).
[25] P. M. Preiss, J. H. Becher, R. Klemt, V. Klinkhamer, A. Bergschneider, and S. Jochim, High-Contrast Interference of Ultracold Fermions, Phys. Rev. Lett. 122, 143602 (2019).

[26] A. Bergschneider, V. M. Klinkhamer, J. H. Becher, R. Klemt, L. Palm, G. Zürn, S. Jochim, and P. M. Preiss, Experimental characterization of two-particle entanglement through position and momentum correlations, Nat. Phys. 15, 640 (2019).

[27] C. Carcy, H. Cayla, A. Tenart, A. Aspect, M. Mancini, and D. Clément, Momentum-Space Atom Correlations in a Mott Insulator, Phys. Rev. X 9, 041028 (2019).

[28] M. Holten, L. Bayha, K. Subramanian, C. Heintze, Ph. M. Preiss, S. Jochim, Observation of Pauli Crystals, Phys. Rev. Lett. 126, 020401 (2021).

[29] L. O. Baksmaty, C. Yannouleas, and U. Landman, Rapidly rotating boson molecules with long- or short-range repulsion: An exact diagonalization study, Phys. Rev. A 75, 023620 (2007).

[30] C. Yannouleas and U. Landman, Third-order momentum correlation interferometry maps for entangled quantal states of three singly trapped massive ultracold fermions, Phys. Rev. A 100 , 023618 (2019).

[31] C. Yannouleas and U. Landman, All-order momentum correlations of three ultracold bosonic atoms confined in triple-well traps. I. Signatures of emergent many-body phase transitions and analogies with three-photon quantum-optics interference, Phys. Rev. A 101, 063614 (2020).

[32] M. Lewenstein, A. Sanpera, V. Ahufinger, B. Damski, A. Sen(De), and U. Sen, Ultracold atomic gases in optical lattices: Mimicking condensed matter physics and beyond, Adv. Phys. 56, 243 (2007).

[33] N. R. Cooper, Rapidly rotating atomic gases, Adv. Phys. 57, 539 (2008)

[34] M. Popp, B. Paredes, and J. I. Cirac, Adiabatic path to fractional quantum Hall states of a few bosonic atoms, Phys. Rev. A 70, 053612 (2004).

[35] S. K. Baur, K. R. A. Hazzard, and E. J. Mueller, Stirring trapped atoms into fractional quantum Hall puddles, Phys. Rev. A 78, 061608(R) (2008).

[36] N. Gemelke, E. Sarajlic, and S. Chu, Rotating few-body atomic systems in the fractional quantum Hall regime, arXiv:1007.2677 (2010).

[37] R. O. Umucalilar, E. Macaluso, T. Comparin, and I. Carusotto, Time-of-Flight Measurements as a Possible Method to Observe Anyonic Statistics, Phys. Rev. Lett. 120, 230403 (2018).

[38] C. Yannouleas and U. Landman, Fractional quantum Hall physics and higher-order momentum correlations in a few spinful fermionic contact-interacting ultracold atoms in rotating traps, Phys. Rev. A 102, 043317 (2020).

[39] L. Palm, F. Grusdt, and P. M. Preiss, Skyrmion ground states of rapidly rotating few-fermion systems, New J. Phys. 22, 083037 (2020).

[40] I. Shavitt, The history and evolution of configuration interaction, Molec. Phys. 94, 3 (1998).

[41] R. B. Laughlin, Anomalous Quantum Hall Effect: An Incompressible Quantum Fluid with Fractionally Charged Excitations, Phys. Rev. Lett. 50, 1395 (1983).

[42] B. I. Halperin, Theory of the quantized Hall conductance, Helv. Phys. Acta 56, 75 (1983).

[43] D. Yoshioka, Wave function of the largest skyrmion on a sphere, J. Phys. Soc. Jpn. 67, 3356 (1998). 
[44] S. H. Simon, Wavefunctionology: The special structure of certain fractional quantum Hall wavefunctions, in Fractional Quantum Hall Effects: New Developments, edited by B. I. Halperin and J. K. Jain (World Scientific, Singapore, 2020).

[45] A. H. MacDonald, H. A. Fertig, and L. Brey, Skyrmions without Sigma Models in Quantum Hall Ferromagnets, Phys. Rev. Lett. 76, 2153 (1996).

[46] J. K. Jain, Composite Fermions (Cambridge University Press, Cambridge, 2007).

[47] N. Rougerie, S. Serfaty, and J. Yngvason, Quantum Hall phases and plasma analogy in rotating trapped Bose gases, J. Stat. Phys. 154, 2 (2014).

[48] E. H. Lieb, N. Rougerie, and J. Yngvason, Rigidity of the Laughlin liquid, J. Stat. Phys. 172, 544 (2018).

[49] R. K. Kamilla, X. G. Wu, and J. K. Jain, Skyrmions in the fractional quantum Hall effect, Solid State Commun. 99, 289 (1996).

[50] See Chap. 11.10. in Ref. [46].

[51] M. Abolfath, J. J. Palacios, H. A. Fertig, S. M. Girvin, and A. H. MacDonald, Critical comparison of classical field theory and microscopic wave functions for skyrmions in quantum Hall ferromagnets, Phys. Rev. B 56, 6795 (1997).

[52] K. Yang, S. Das Sarma, and A. H. MacDonald, Collective modes and skyrmion excitations in graphene $S U(4)$ quantum Hall ferromagnets, Phys. Rev. B 74, 075423 (2006).

[53] S. M. Girvin, The quantum Hall effect: Novel excitations and broken symmetries, in Lectures delivered at École d' Été Les Houches, July 1998, Topological Aspects of Low Dimensional Systems, edited by A. Comtet, T. Jolicoeur, S. Ouvry, and F. David (Springer-Verlag, Berlin, and Les Éditions de Physique, Les Ulis, 2000); S. M. Girvin and A. H. MacDonald, Multicomponent quantum Hall systems: The sum of their parts and more, in Perspectives in Quantum Hall Effects: Novel Quantum Liquids in Low-Dimensional Semiconductor Structures, edited by S. Das Sarma and A. Pinczuk (Wiley-VCH, 1996), pp.161-224.

[54] C. Yannouleas and U. Landman, Trial wave functions with longrange Coulomb correlations for two-dimensional $\mathrm{N}$-electron systems in high magnetic fields, Phys. Rev. B 66, 115315 (2002).

[55] C. Yannouleas and U. Landman, Two-dimensional quantum dots in high magnetic fields: Rotating-electron-molecule versus composite-fermion approach, Phys. Rev. B 68, 035326 (2003).

[56] C. Yannouleas and U. Landman, Structural properties of electrons in quantum dots in high magnetic fields: Crystalline character of cusp states and excitation spectra, Phys. Rev. B 70, 235319 (2004).

[57] C. Yannouleas and U. Landman, Symmetry breaking and quantum correlations in finite systems: Studies of quantum dots and ultracold Bose gases and related nuclear and chemical methods, Rep. Prog. Phys. 70, 2067 (2007).

[58] I. Romanovsky, C. Yannouleas, L. O. Baksmaty, and U. Landman, Bosonic Molecules in Rotating Traps, Phys. Rev. Lett. 97, 090401 (2006).

[59] See Supplemental Material at http://link.aps.org/supplemental/ 10.1103/PhysRevResearch.3.L032028 for (1) A detailed com- parison with the symmetric polynomials for skyrmions in earlier literature; (2) Additional specific examples of the compact analytic wave functions; (3) Tables illustrating, for $N=4$ and $N=9$, the correspondence between numerical CI and algebraic coefficients in the determinantal expansions of $\Phi_{\mathrm{CI}}$ and $\Phi_{\mathrm{alg}}$; (4) Figure displaying additional higher-order correlations for $N=9$ LLL fermions.

[60] Spinful Jack polynomials have been studied for the singlet ( $S=S_{z}=0$ ) state only; see, e.g., B. Estienne and A. Bernevig, Spin-singlet quantum Hall states and Jack polynomials with a prescribed symmetry, Nucl. Phys. B 857, 185 (2012); S. C. Davenport, E. Ardonne, N. Regnault, and S. H. Simon, Spinsinglet Gaffnian wave function for fractional quantum Hall systems, Phys. Rev. B 87, 045310 (2013). The singlet trial functions employed in Refs. [39,43] are in agreement with the exact analytic expressions derived here.

[61] M. Roncaglia, M. Rizzi, and J. Dalibard, From rotating atomic rings to quantum Hall states, Sci. Rep. 1, 43 (2011).

[62] F. D. M. Haldane, Fractional Quantization of the Hall Effect: A Hierarchy of Incompressible Quantum Fluid States, Phys. Rev. Lett. 51, 605 (1983).

[63] X.-G. Wen, Theory of the edge states in fractional quantum Hall effects, Int. J. Mod. Phys. B 6, 1711 (1992).

[64] Wolfram Research, Inc., Mathematica, Version 12.1, Champaign, IL (2020).

[65] R. B. Lehoucq, D. C. Sorensen, and C. Yang, ARPACK Users Guide: Solution of Large-Scale Eigenvalue Problems with Implicitly Restarted Arnoldi Methods (SIAM, Philadelphia, 1998).

[66] W. E. Arnoldi, The principle of minimized iterations in the solution of the matrix eigenvalue problem, Q. Appl. Math. 9 , 17 (1951)

[67] J. H. van Vleck, Nonorthogonality and ferromagnetism, Phys. Rev. 49, 232 (1936).

[68] P. W. Anderson, Infrared Catastrophe in Fermi Gases with Local Scattering Potentials, Phys. Rev. Lett. 18, 1049 (1967).

[69] W. Kohn, Nobel Lecture: Electronic structure of matter-wave functions and density functionals, Rev. Mod. Phys. 71, 1253 (1999).

[70] M. Knap, A. Shashi, Y. Nishida, A. Imambekov, D. A. Abanin, and E. Demler, Time-Dependent Impurity in Ultracold Fermions: Orthogonality Catastrophe and Beyond, Phys. Rev. X 2, 041020 (2012).

[71] J. R. McClean, R. Babbush, P. J. Love, and A. Aspuru-Guzik, Exploiting locality in quantum computation for quantum chemistry, J. Phys. Chem. Lett. 5, 4368 (2014).

[72] F. Ares, K. S. Gupta, and A. R. de Queiroz, Orthogonality catastrophe and fractional exclusion statistics, Phys. Rev. E 97 022133 (2018).

[73] J. Gu, Anderson orthogonality catastrophe in 2 + 1-D topological systems, arXiv:1905.00171 (2019).

[74] V. A. Fock, Wave functions of many-electron systems, J. Exp. Theo. Phys. (U. S. S. R.) 10, 961 (1940).

[75] E. Macaluso and I. Carusotto, Hard-wall confinement of a fractional quantum Hall liquid, Phys. Rev. A 96, 043607 (2017). 